\title{
Magnetar X-ray emission mechanisms
}

\section{Silvia Zane}

\author{
Mullard Space Science Laboratory, University College London, United Kingdom \\ email: sz@mssl.ucl.ac.uk
}

\begin{abstract}
Soft gamma-ray repeaters (SGRs) and anomalous X-ray pulsars (AXPs) are peculiar $\mathrm{X}$-ray sources which are believed to be magnetars: ultra-magnetized neutron stars which emission is dominated by surface fields (often in excess of $1 \mathrm{E} 14 \mathrm{G}$, i.e. well above the QED threshold).

Spectral analysis is an important tool in magnetar astrophysics since it can provide key information on the emission mechanisms. The first attempts at modelling the persistent (i.e. outside bursts) soft X-ray ( $\mathrm{i} 10 \mathrm{keV})$ spectra of AXPs proved that a model consisting of a blackbody (kT $0.3-0.6 \mathrm{keV}$ ) plus a power-law (photon index 2-4) could successfully reproduce the observed emission. Moreover, INTEGRAL observations have shown that, while in quiescence, magnetars emit substantial persistent radiation also at higher energies, up to a few hundreds of $\mathrm{keV}$. However, a convincing physical interpretation of the various spectral components is still missing.

In this talk I will focus on the interpretation of magnetar spectral properties during quiescence. I will summarise the present status of the art and the currents attempts to model the broadband persistent emission of magnetars (from IR to hard Xrays) within a self consistent, physical scenario.
\end{abstract}

